\title{
METRIC SPACES ALL OF WHOSE DECOMPOSITIONS ARE METRIC
}

\section{STEPHEN WILLARD}

1. Introduction. This note consists of some consequences of the following theorem of Hanai and Morita [2] and Stone [4].

Theorem. If $f: X \rightarrow Y$ is closed, continuous and onto and $X$ is metric, the following are equivalent:

(1) $Y$ is metric.

(2) $Y$ is first countable.

(3) For each $p \in Y, f^{-1}(p)$ has compact frontier.

In particular, we use this result to obtain a list of characterizations of those metric spaces $X$ each of whose closed, continuous images is metric (Theorem 1), the most interesting being that the only such spaces are simple combinations of compact spaces and discrete spaces. We then apply this characterization to derive the converse to the well-known result that every Hausdorff continuous image of a compact metric space is metrizable; i.e., if every Hausdorff continuous image of a metric space $X$ is metrizable, then $X$ is compact (Theorem 3).

2. $A$-spaces. We will call a metric space $X$ an $A$-space iff every Hausdorff image of $X$ under a closed continuous map is metrizable. By the theorem above, then, $X$ is an $A$-space iff every closed set in $X$ has compact frontier. To develop our characterization theorem, we need some notation.

Let $F$ be a nonempty closed subset of $X, Y$ the space obtained from $X$ by identifying the points of $F$, with $h: X \rightarrow Y$ the identification map. Let $\beta X$ be the Stone-Cech compactification of $X$ and $K$ a closed subset of $\beta X$ such that (1) $K \cap X=F$, and (2) whenever $U$ is open in $X$ and $U \supset F$, then $U=U_{0} \cap X, U_{0}$ open in $\beta X, U_{0} \supset K$ (e.g., $\left.K=\mathrm{Cl}_{\beta X} F\right)$. Denote by $Y_{K}$ the space obtained from $\beta X$ by identifying the points of $K$, and by $H_{K}$ the corresponding identification map. Then $Y_{K}$ will be a compactification of $Y$ (conditions (1) and (2) above ensure that $Y_{K}$ will contain $Y$ set-theoretically and topologically, respectively), and $H_{K} \mid X=h$. Among these compactifications, if $K=\mathrm{Cl}_{\beta X} F$, then $Y_{K}=\beta Y$.

We are ready for our first equivalence.

Received by the editors August 5, 1966 and, in revised form, March 5, 1968. 
THEOREM 1. The following are equivalent for a metric space $X$ :

(a) $X$ is an $A$-space.

(b) $\mathrm{Cl}_{\beta X} F$ is a zero-set in $\beta X$ whenever $F$ is a zero-set (i.e., is closed) in $X$.

(c) $K$ is a zero-set in $\beta X$ whenever $K$ is closed in $\beta X$ and has the property that, for every open set $U$ of $X$ containing $X \cap K$, there is an open set $U_{0}$ in $\beta X$ such that $U_{0} \supset K$ and $U_{0} \cap X=U$.

(d) $X=X_{0} \cup D$, where $X_{0}$ is compact and $D$ consists of points isolated in $X$.

Proof. (a) $\Rightarrow$ (c): Let $X$ be an $A$-space, let $K$ be as in (c) and put $F=X \cap K$. We may assume $F \neq \varnothing$ (else $K=\varnothing$, a zero-set), so the remarks above apply. That is, $Y_{K}$ is a compactification of the space $Y$ obtained by identifying the points of $F$, and $Y$ is metric since $X$ is an $A$-space. Hence, $p=h(F)=H_{K}(K)$ is 1st countable in $Y$, and thus in $Y_{K}$. Hence, $H_{K}^{-1}(p)=K$ is a closed $G_{\delta}$ set in $\beta X$, i.e. $K$ is a zero-set.

(c) $\Rightarrow$ (b): Trivial, since $\mathrm{Cl}_{\beta X} F$ satisfies the properties of $K$ in (c).

(b) $\Rightarrow$ (a): Let $F$ be any closed set in $X, h: X \rightarrow Y$ the map identifying $F$ to a point $p$. Now, by (b), $\mathrm{Cl}_{\beta X} F=\bigcap_{n=1}^{\infty} V_{n}$, each $V_{n}$ open in $\beta X$. But, setting $K=\mathrm{Cl}_{\beta X} F$, we have $Y_{K}=\beta Y$, and since $H_{K}$ is oneone outside $K, H_{K}\left(\mathrm{Cl}_{\beta X} F\right)=p=\bigcap_{n=1}^{\infty} H_{K}\left(V_{n}\right)$, so $p$ is a $G_{\delta}$ in the compact space $\beta Y$ and hence has a countable base in $\beta Y$. Thus, $p$ has a countable base in $Y$. It follows from the Hanai-Morita-Stone Theorem that $F$ has compact frontier. Thus, $X$ is an $A$-space.

(a) $\Rightarrow$ (d): Suppose $X$ is an $A$-space. Let $D$ be the set of all points isolated in $X$. Then $X_{0}=X-D$ is closed in $X$. We will show it is compact. If not, let $F=\left\{x_{1}, x_{2}, \cdots\right\}$ be a sequence in $X_{0}$ having no cluster point in $X_{0}$. Clearly, $F=\operatorname{Fr}(F) \subset X_{0}$, and it follows that $\operatorname{Fr}(F)$ is not compact-contradicting the assumption that $X$ is an $A$-space.

(d) $\Rightarrow(\mathrm{a})$ : Let $F$ be closed in $X$. If $x \in D$, then $x \notin \operatorname{Fr}(F)$. Thus $\operatorname{Fr}(F) \subset X_{0}$, so $\operatorname{Fr}(F)$ is compact. Hence, $X$ is an $A$-space.

This completes the proof of Theorem 1 .

Our main interest is in the characterization (d) of $A$-spaces. It shows that the $A$-spaces are identical with spaces studied by Nagata [3], Atsuji [1] and Stone [4] in other contexts. Nagata showed that the $A$-spaces are completely metrizable by constructing an explicit complete metric. We can give a quick proof of the existence of complete metrics by turning to the metric completion $X^{*}$ of $X$.

Theorem 2. If $X$ is an A-space, then $X$ has a complete metric (i.e., is completely metrizable as a topological space). 
Proof. If $X$ is an $A$-space, then $X=X_{0} \cup D$, where $X_{0}$ is compact, $D$ consists of isolated points and $X_{0} \cap D=\varnothing$. If $X^{*}$ is the metric completion of $X$, then each point of $D$ is isolated in $X^{*}$ (by a diagonal process, if a point of $D$ is a limit of points from $X^{*}$, it will be a limit of points from $X$ ), so $D$ is open in $X^{*}$. Since $X_{0}$ is closed in $X^{*}$, and thus a $G_{\delta}$ in $X^{*}$, it follows that $X=X_{0} \cup D$ is a $G_{\delta}$ in $X^{*}$, and thus completely metrizable.

3. Continuous images in general. Making use of Theorem 1 , we can now provide an easy proof to the converse of the well-known result that every Hausdorff continuous image of a compact metric space is metrizable.

THEOREM 3. If $X$ is a metric space such that every Hausdorff continuous image of $X$ is metrizable, then $X$ is compact.

Proof. If $X$ is such a space, then $X$ is an $A$-space, so $X=X_{0} \cup D$ where $X_{0}$ is compact and $D$ consists of the isolated points of $X$.

Suppose a sequence $x_{1}, x_{2}, \cdots$ of points of $D$ can be found such that $\left\{x_{1}, x_{2}, \ldots\right\}$ has no cluster point in $X$. Construct a new space $Y$ from $X$ as follows: Retain the old structure on $X-\left\{x_{1}, x_{2}, \ldots\right\}$ and on $\left\{x_{1}, x_{2}, \cdots\right\}$ put any Hausdorff nonmetric topology, letting $Y$ be the disjoint union of the spaces $X-\left\{x_{1}, x_{2}, \cdots\right\}$ and $\left\{x_{1}, x_{2}, \cdots\right\}$ thus obtained. Then $Y$ is a continuous Hausdorff image of $X$ (under the identity) but $Y$ is not metric. Impossible. Thus every sequence $\left\{x_{1}, x_{2}, \cdots\right\}$ of points of $D$ has a cluster point in $X$.

Now if $\mathcal{u}$ is any open cover of $X$, a finite subcollection $\left\{U_{1}, \cdots, U_{n}\right\}$ from $\mathcal{u}$ covers $X_{0}$, and, from above, there can be only finitely many points of $D$ outside $U_{1} \cup \ldots \cup U_{n}$. Thus a finite subcollection from $u$ will cover $X$.

Thus $X$ itself is compact.

\section{REFERENCES}

1. M. Atsuji, Uniform continuity of continuous functions of metric spaces, Pacific J. Math. 8 (1958), 11-16.

2. S. Hanai and K. Morita, Closed mappings and metric spaces, Proc. Japan Acad. 32 (1956), 10-14.

3. J. Nagata, On the uniform topology of biocompactifications, J. Inst. Polytech. Osaka City Univ. Ser. A Math. 1 (1950), 28-38.

4. A. H. Stone, Metrizability of decomposition spaces, Proc. Amer. Math. Soc. 7 (1956), 690-700.

5. - Universal spaces for some metrizable uniformities, Quart. J. Math. Oxford Ser. (2) 11 (1960), 105-115.

Case Western Reserve University 